\title{
Caracterización del rol actual de la mujer en la gerencia de proyectos en Colombia
}

\author{
Fredy Carreño Sánchez ${ }^{1}$ \\ Escuela Colombiana de Ingeniería Julio Garavito \\ fredy.carreno@escuelaing.edu.co \\ Ángela C. Vargas Marín ${ }^{2}$ \\ Escuela Colombiana de Ingeniería Julio Garavito \\ angela.vargas-ma@mail.escuelaing.edu.co \\ Germán Eduardo Giraldo ${ }^{3}$ \\ Escuela Colombiana de Ingeniería Julio Garavito \\ german.giraldo@escuelaing.edu.co \\ DOI: https://doi.org/10.21158/01208160.n83.2017.1823
}

Fecha de recepción: 19 de julio de 2017

Fecha de aprobación: 12 de septiembre de 2017

Cómo citar este artículo / To reference this article / Comment citer cet article / Para citar este artigo:

Carreño, F., Vargas, A. y Giraldo, G. 2017). Caracterización del rol actual de la mujer en la gerencia de proyectos en Colombia. Revista EAN, 83, PP 93-117. https://doi.org/10.21158/01208160.n83.2017.1823

\section{Resumen}

En este estudio, se identifican características de mujeres que desempeñan roles de gerencia de proyectos en Colombia, en general, información sobre sus competencias, habilidades, capacitación, entre otras, a partir de las cuales se puedan proponer acciones que contribuyan a incrementar la participación y a mejorar su desempeño en esta disciplina. El estudio se realizó a partir de la revisión de literatura con información sobre estándares de competencias y habilidades de los gerentes de proyectos y de resultados de investigaciones en torno al tema, y la participación de mujeres en la gerencia de proyectos. Para este estudio, de enfoque cualitativo, se aplicó un cuestionario estructurado en línea, respondido por 21 participantes. A partir de la experiencia, se proponen acciones en etapas posteriores de la investigación y en trabajos futuros.

\section{Palabras clave}

Caracterización, rol, mujer, gerente de proyectos, gerencia de proyectos.

\footnotetext{
${ }^{1}$ Ingeniero civil de la Escuela Colombiana de Ingeniería Julio Garavito. Especialista y magíster en Desarrollo y Gerencia Integral de Proyectos de la Escuela Colombiana de Ingeniería Julio Garavito. Profesor de planta Escuela Colombiana de Ingeniería Julio Garavito. ORCID: http://orcid.org/0000-0002-7067-7151

${ }^{2}$ Magíster en Desarrollo y Gerencia Integral de Proyectos de la Escuela Colombiana de Ingeniería Julio Garavito. Especialista de soporte técnico de tecnologías de protección DuPont do Brasil.

${ }^{3}$ Ingeniero industrial de la Universidad de la Sabana. Magíster en Metodología para la reorganización perpetua de almacenes de la Universidad de Puerto Rico. Profesor de planta Escuela Colombiana de Ingeniería Julio Garavito.
} 


\title{
Characterization of the current role of women in project management in Colombia
}

\begin{abstract}
This research study aims at identifying the characteristics of women who play the role of project managers in Colombia, specifically giving information about competences, skills, training, among others, out of which different actions are suggested to increase participation and to improve women's performance in this specific field. The research was made starting with bibliographic revision on the standards of project managers' competences and skills and its resulting research outcomes about this specific topic as well as the participation of women in project management. For this research study focusing on a qualitative approach, an online structured questionnaire was applied, being answered by twenty one participants. Based on this experience, different actions were proposed through different steps following this investigation and other future studies.
\end{abstract}

Key words. Characterization, role, women, project manager, project management.

\section{Caractérisation du rôle actuel des femmes dans la gestion de projets en Colombie}

Résumé. Cette étude tente d'identifier les caractéristiques des femmes occupant des postes à responsabilités dans le domaine de la gestion de projets en Colombie en présentant des informations sur leurs compétences et leurs formations et proposant des actions destinées à améliorer la participation et la performance des femmes dans ce domaine d'activité. L'étude présente également une analyse de la littérature existante sur le sujet, informe sur les standards en termes de compétences des gestionnaires de projets, donne des résultats d'investigations et présente la participation des femmes dans le domaine de la gestion de projets. Pour réaliser cette investigation de type qualitative, vingt et une réponses à un questionnaire en ligne ont été analysées permettant de proposer des solutions et de futures actions.

Mots clefs. Caractérisation, rôle, femme, gestionnaire de projets, gestion de projets.

\section{Caracterização do papel atual da mulher na gerência de projetos na Colômbia}

Resumo.Neste estudo, identificam-se as características gerais das mulheres que desempenham papéis de gerência de projetos na Colômbia, informações sobre suas competências, habilidades, capacitação, entre outras, a partir das quais se podem propor ações que contribuam para aumentar a participação e a melhora do desempenho das mulheres nesta disciplina. O estudo se realizou a partir da literatura com informações sobre padrões de competências $e$ habilidades dos gerentes de projetos, a partir dos resultados de pesquisas relacionadas ao assunto e a partir da participação das mulheres no gerenciamento de projetos. Para este estudo, de enfoque qualitativo, foi aplicado um questionário estruturado on-line, respondido por vinte e um participantes. Com base na experiência, são propostas ações em etapas posteriores da pesquisa e em trabalhos futuros.

Palavras-chave. Gerenciamento de projetos, gerente de projeto, mulher, caracterização, papel. 


\section{Introducción}

T a gerencia moderna de proyectos se Lencuentra presente en todos los sectores de la economía nacional y ha sido reconocida en el ámbito empresarial como «la nueva forma de administraciónı, siendo la encargada de materializar los objetivos estratégicos y dado su vital aporte al logro de la visión de cualquier tipo de organización (Giraldo, Pulido y Leal, 2013).

Actualmente, no se cuenta con información formal sobre el rol de la mujer en la gerencia de proyectos en Colombia y menos aún sobre las habilidades requeridas para llevar a cabo una adecuada gestión al frente de un proyecto. Disponer de la caracterización de las mujeres como gerentes de proyectos, incluso información sobre las habilidades requeridas para desempeñar este rol, permitirá proponer y desarrollar herramientas que permitan mejorar su desempeño en el área, y por tanto, incrementar su participación en este campo laboral.

En elámbitointernacional, seha empezado a analizar la presencia de la mujer en la gerencia de proyectos y se evidencia aumento en el número de mujeres que se forman y se vinculan a grupos de interés relacionados con el tema (Burba, 2010). Sin embargo, su participación en este rol dentro del mercado laboral no ha cambiado significativamente desde 2007 (Botkova, 2008). De acuerdo con el International Business Report (Grant Thornton, 2014), globalmente, solo $24 \%$ de los cargos gerenciales son ocupados por mujeres, y en países como Alemania, solo $33 \%$ de las empresas cuenta con mujeres en posiciones gerenciales. Así mismo, los hombres continúan siendo la fuerza dominante en la gerencia de proyectos, particularmente en áreas relacionadas con la ingeniería, tales como construcción, informática, telecomunicaciones y proyectos aeroespaciales, entre otros (Botkova, 2008).

De acuerdo con el estudio realizado por Neuhauser (2007), se destacan cinco comportamientos frecuentes en mujeres a cargo de la gerencia de proyectos: $\mathrm{i}$ ) recomendar ascensos por desempeño excepcional; ii) recomendar aumentos en remuneración o pago de bonificaciones por desempeño excepcional; iii) delegar autoridad decisoria a miembros de su equipo; iv) tomar ideas aceptadas por sus superiores; y v) que la gente se enorgullezca de ser asociada con ellas. En el mismo estudio, se destacan cinco comportamientos infrecuentes: i) obtener el respeto de otros al demostrar sus competencias en el desempeño personal; ii) inspirar a otros siendo ejemplo de coraje, dedicación y sacrificio; iii) usar el tiempo eficientemente; iv) negociar con colegas, proveedores y clientes, y v) ofrecer compromiso, consejo y apoyo a miembros del equipo cuando lo requieran.

En su tesis de doctorado, Donna M. Karch cuantificó el desempeño de las mujeres en comportamientos específicos necesarios para la gerencia de proyectos comparado con el desempeño de los hombres, y encontró pequeñas diferencias en la percepción de la importancia de dichos comportamientos y en su uso para el mismo fin (Karch, 2012).

Este artículo se desarrolla con el fin de realizar la caracterización del rol actual de la mujer en la gerencia de proyectos en Colombia, y se lleva a cabo como parte del proyecto de investigación de «Identificación, caracterización y análisis de la mujer en el rol de gerente de proyectos en Colombialy, que lleva a cabo el Centro de Estudios en 
Desarrollo y Gerencia Integral de Proyectos de la Escuela Colombiana de Ingeniería Julio Garavito.

La investigación contempla una revisión documental respecto de la mujer en roles gerenciales, particularmente en el de gerente de proyectos; así mismo, sobre las habilidades y competencias que requiere un gerente de proyectos para poder desempeñar adecuadamente su rol.

Se aborda un análisis cualitativo del perfil de la mujer en la gerencia de proyectos en Colombia, realizado según la aplicación de un cuestionario estructurado que permitirá determinar diferencias desde la perspectiva de quienes participan en la encuesta, entre las condiciones a las cuales se enfrentan las mujeres gerentes de proyectos en el campo laboral, comparadas con aquellas que enfrentan sus pares hombres. Se estudian aspectos generales de la situación de las encuestadas, como su entorno familiar, formación y remuneración; su trayectoria profesional respecto de las funciones, niveles de autoridad y habilidades propias de su perfil; su rol y su desempeño como gerentes de proyectos dentro de las organizaciones a las cuales pertenecen. Se estudian aspectos de percepción sobre su posición en la organización respecto de sus pares hombres y la percepción de diferencias en el desempeño de los proyectos referidos a aquellos liderados por hombres. Finalmente, se presentan conclusiones y recomendaciones frente a la investigación realizada, con miras a continuar el proceso de análisis dentro del proyecto macro.

\section{Marco teórico}

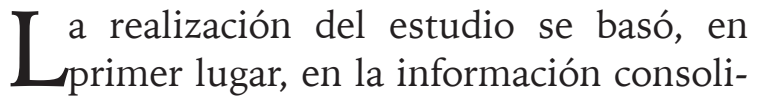
dada sobre las competencias encontradas en la revisión de la literatura que se requieren para que un gerente de proyectos desempeñe satisfactoriamente su rol; y en segundo lugar, en los resultados de investigaciones realizadas tanto en Colombia como en otros países acerca de la participación de mujeres en roles gerenciales, particularmente en el de la gerencia de proyectos.

\subsection{Habilidades interpersonales}

Las habilidades interpersonales cobran cada vez mayor importancia en el desarrollo de los proyectos dada la presión que se tiene en el mundo competitivo de hoy, la necesidad de trabajar con equipos globalizados, la complejidad de los proyectos, la cantidad de interesados, la naturaleza matricial de las organizaciones y la falta de clara definición de roles, entre otras razones (Levin, 2010). Algunas de las habilidades consideradas como fundamentales en la gerencia de proyectos se describen a continuación. 


\subsubsection{Liderazgo}

Se entiende como la capacidad que tiene un gerente para lograr que el equipo realice el trabajo necesario para alcanzar los objetivos y el éxito del proyecto. De acuerdo con la Guía del PMBOK ${ }^{\circledR}$, el liderazgo eficaz debe estar basado en el respeto y la confianza más que en el miedo y la sumisión (PMI, 2013a). Para completar un proyecto a tiempo y dentro del presupuesto, de acuerdo con las especificaciones y lograr la satisfacción del cliente, un gerente de proyectos debe ser líder, gestor, facilitador y mentor (Levin, 2010), cuatro roles importantes, propios del liderazgo necesarios para alcanzar el éxito en los proyectos. Según Crowe, «los buenos gerentes entregan resultados mientras que los buenos líderes desarrollan personas, y en la mayoría de las organizaciones, los gerentes exitosos de proyectos deben ser capaces de lograr ambas $\iota^{4}$ (Crowe, 2008).

\subsubsection{Desarrollo del espíritu de equipo}

A través del entendimiento común de los objetivos de un proyecto, se orienta a los miembros del equipo hacia el trabajo conjunto, incluso a los interesados y a la organización (PMI, 2013a). Las actividades para el desarrollo del espíritu de equipo incluyen el establecimiento de objetivos, la negociación y definición de roles y la implantación de procesos. Es un ejercicio continuo que debe llevarse a cabo a lo largo del ciclo de vida de un proyecto (Levin, 2010).

\subsubsection{Motivación}

Kerzner y Salidis (2009), citados en Levin (2010), definen la motivación como el «alentar a otros a desempeñarse mediante el cumplimiento o apelando a sus propias necesidadesı); es decir, lograr que las personas realicen el trabajo por la satisfacción del deber cumplido o a través de incentivos. Una clave está en la capacidad de enfocar las situaciones a las condiciones propias de cada miembro del equipo de acuerdo con sus necesidades, incluso, la satisfacción profesional, la sensación de realización y la adecuada compensación financiera, entre otros aspectos.

\subsubsection{Comunicación}

La buena gerencia de proyectos implica la necesidad de establecer con claridad los requerimientos y los estilos de comunicación de los interesados, incluso a los miembros del equipo, y el uso apropiado de las herramientas y los canales disponibles (PMI, 2013a).

\subsubsection{Toma de decisiones}

Un gerente de proyectos dispone de cuatro estilos básicos para la toma de decisiones: i) ordenar ii) consultar iii) consensuar, y iv) aleatorio. En general, esta puede ser afectada por las restricciones de tiempo, la confianza, la calidad y la aceptación (PMI, 2013a).

\footnotetext{
${ }^{4}$ Las traducciones son nuestras.
} 


\subsubsection{Negociación}

«Es un proceso deliberado entre dos o más partes, en la búsqueda de la solución de una situación común» (Harvard Law School, 2015). Como habilidad incrementa las probabilidades de éxito de un proyecto. De acuerdo con la Guía del $\mathrm{PMBOK}^{\circledR}$, en una negociación exitosa se debe analizar la situación, diferenciar entre necesidades y deseos, centrarse en los intereses, escuchar atentamente y comunicarse fluidamente, de tal forma que todas las partes se sientan favorecidas (PMI, 2013a).

\subsubsection{Gestión de conflictos}

Por diversas razones los conflictos resultan en forma inevitable en cualquier proyecto. Una gestión eficaz de conflictos permite minimizar los posibles impactos negativos, de forma que el equipo pueda plantear mejores soluciones que aumenten la probabilidad de éxito del proyecto (PMI, 2013a). Según Levin (2010), un conflicto puede resolverse a través de diferentes enfoques, como la suavización, la competencia o presión, la evitación, el compromiso, la confrontación la colaboración, entre otros, dependiendo de las circunstancias.

\subsubsection{Estándares internacionales sobre competencias para la gerencia de proyectos}

Las organizaciones internacionales que estudian la disciplina de la gerencia de proyectos y contribuyen a su desarrollo incluyen dentro de sus propuestas estándares sobre las competencias, habilidades y características con las que debería contar un gerente de proyectos para un adecuado desempeño. Las más destacadas de estas organizaciones son el Project Management Institute (PMI) y la International Project Management Association (IPMA).

\subsubsection{PMCD Framework}

El Project Management Competency Development Framework (PMI, 2007) es el estándar en que el PMI plantea dimensiones clave de competencias tanto de desempeño como personales, con importantes impactos positivos en el rol de los gerentes de proyectos. Se entiende como competencias de desempeño aquellas adquiridas a través del conocimiento, mientras que las competencias personales son los comportamientos, las actitudes y las características que contribuyen a la habilidad de una persona para ser gerente de proyectos. Un poco más adelante (Tabla 1 ), se resumen las competencias personales agrupadas en sus respectivas unidades. 
Tabla 1. Unidades de competencias personales según el PMCD Framework, $2^{\mathrm{a}}$ edición.

\begin{tabular}{|l|c|l|}
\hline Unidad de competencias & Página & \multicolumn{1}{|c|}{ Competencias } \\
\hline Comunicación & $26-27$ & $\begin{array}{l}\text { Escuchar activamente, entender y dar respuesta a interesados, } \\
\text { mantener líneas de comunicación, asegurar la calidad de la } \\
\text { información, adaptar comunicaciones. }\end{array}$ \\
\hline Liderazgo & $28-29$ & $\begin{array}{l}\text { Crear ambiente de trabajo en equipo, construir y mantener relaciones } \\
\text { efectivas, motivar y dar tutoría a los miembros del equipo, asumir } \\
\text { responsabilidad por los entregables, usar herramientas para influir. }\end{array}$ \\
\hline Gerencia & $30-31$ & $\begin{array}{l}\text { Construir y mantener el equipo del proyecto, planear y manejar el } \\
\text { desarrollo del proyecto, resolver conflictos. }\end{array}$ \\
\hline Habilidades cognitivas & $32-33$ & $\begin{array}{l}\text { Desarrollar una visión holística del proyecto, solucionar efectivamente } \\
\text { problemas, utilizar apropiadamente herramientas y técnicas de } \\
\text { gerencia de proyectos, buscar oportunidades para mejorar los } \\
\text { resultados del proyecto. }\end{array}$ \\
\hline Efectividad & $34-35$ & $\begin{array}{l}\text { Resolver problemas, mantener motivación, compromiso y apoyo de } \\
\text { los interesados Actuar oportunamente frente a los cambios, usar } \\
\text { asertividad. }\end{array}$ \\
\hline Profesionalismo & $36-38$ & $\begin{array}{l}\text { Demostrar compromiso con el proyecto, operar con integridad, } \\
\text { manejar las adversidades del personal del equipo, dirigir fuerzas de } \\
\text { trabajo diversas, solucionar problemas individuales y organizacionales. }\end{array}$ \\
\hline
\end{tabular}

Fuente. Elaboración propia.

\subsubsection{IPMA Competence Baseline}

La IPMA es una federación conformada por más de 55 asociaciones de diferentes lugares del mundo, las cuales en sus respectivas áreas de influencia aportan al desarrollo de competencias para la gerencia de proyectos, programas y portafolios a través de la interacción entre profesionales, y de sus relaciones con empresas, agencias gubernamentales, universidades y consultoras (IPMA, 2015). Uno de los principales estándares de la IPMA es la IPMA Competence Baseline-ICB, versión 3.0 (IPMA, 2006), en la cual se proponen 46 elementos de competencias para la gerencia de proyectos agrupadas en veinte competencias técnicas, 15 competencias de comportamiento y 11 competencias contextuales que se integran en una visión global conocida como The Eye of Competence.

\subsubsection{The Australian Institute of Project Management (AIPM)}

El AIPM es la principal institución para gerencia de proyectos en Australia. Así mismo, es parte activa de la IPMA y procura por la mejora en el conocimiento, las habilidades y las competencias de los gerentes y miembros de equipos de proyectos (AIPM, 2015). El AIPM publicó, en 2008, las más recientes versiones de sus estándares de competencias profesionales para la gestión de proyectos divididos en cuatro partes. Específicamente la parte C, conocida como Certified Practising Project Manager (CPPM) (AIPM, 2010), y que hace referencia a la gerencia de proyectos, presenta las habilidades que debe tener un gerente en cada una de las nueve unidades de la gestión de proyectos.

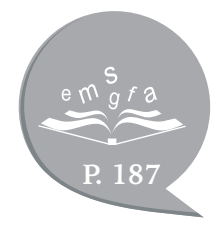


Tabla 2. Competencias para la gerencia de proyectos según el ICB® de la IPMA

\begin{tabular}{|l|c|l|}
\hline Ámbito de competencias & Páginas & \multicolumn{1}{|c|}{ Elementos de competencias } \\
\hline Competencias técnicas & $38-81$ & $\begin{array}{l}\text { Éxito en la gerencia de proyectos, partes interesadas, requisitos } \\
\text { y objetivos de proyectos, riesgos y oportunidades, calidad, } \\
\text { organizaciones de proyectos, trabajo en equipo, resolución de } \\
\text { problemas, estructuras de proyectos, alcance y entregables, tiempo y } \\
\text { fases de proyectos, recursos, costo y financiación, aprovisionamiento } \\
\text { y contratos, cambios, controles e informes, información y } \\
\text { documentación, comunicación, puesta en marcha, cierre. }\end{array}$ \\
\hline Competencias de comportamiento & $83-124$ & $\begin{array}{l}\text { Liderazgo, compromiso y motivación, autocontrol, confianza en } \\
\text { sí mismo, relajación, actitud abierta, creatividad, orientación } \\
\text { a resultados, eficiencia, consulta, conflictos y crisis, fiabilidad, } \\
\text { apreciación de valores, ética. }\end{array}$ \\
\hline Competencias contextuales & $125-154$ & $\begin{array}{l}\text { Orientación a proyectos, orientación a programas, orientación a } \\
\text { portafolios, implantación de proyectos, programas y portafolios, } \\
\text { organizaciones permanentes, negocios, sistemas, productos y } \\
\text { tecnologías, dirección de personal, seguridad, higiene y medio } \\
\text { ambiente, finanzas. }\end{array}$ \\
\hline
\end{tabular}

Fuente. Elaboración propia.

Tabla 3. Competencias para la gerencia de proyectos según el CPPM $®$ del AIPM

\begin{tabular}{|c|c|c|}
\hline Unidad & Página & Habilidades \\
\hline Planear, gestionar y revisar el alcance. & 5 & $\begin{array}{l}\text { Conocimiento práctico sobre el negocio, planeación y organización, } \\
\text { resolución de problemas, análisis, negociación y gestión del } \\
\text { cambio. }\end{array}$ \\
\hline Planear, gestionar y revisar el tiempo. & 8 & $\begin{array}{l}\text { Conocimiento práctico, autogestión, organización, comunicación, } \\
\text { habilidades tecnológicas, análisis. }\end{array}$ \\
\hline Planear, gestionar y revisar el costo. & 11 & $\begin{array}{l}\text { Cálculo y habilidades en presupuestos, análisis, habilidades } \\
\text { tecnológicas, Conocimiento práctico. }\end{array}$ \\
\hline Planear, gestionar y revisar la calidad. & 14 & Conocimiento práctico, comunicación, análisis. \\
\hline $\begin{array}{l}\text { Planear, gestionar y revisar los } \\
\text { recursos humanos del proyecto. }\end{array}$ & 17 & $\begin{array}{l}\text { Planeación, comunicación, liderazgo, habilidades interpersonales, } \\
\text { instrucción, tutoría, análisis. }\end{array}$ \\
\hline $\begin{array}{l}\text { Planear, gestionar y revisar las } \\
\text { comunicaciones. }\end{array}$ & 21 & $\begin{array}{l}\text { Conocimiento práctico, habilidades tecnológicas, organización, } \\
\text { análisis. }\end{array}$ \\
\hline $\begin{array}{l}\text { Planear, gestionar y revisar el riesgo } \\
\text { del proyecto. }\end{array}$ & 24 & $\begin{array}{l}\text { Conocimiento práctico, comunicación, resolución de problemas, } \\
\text { pensamiento lateral, planeación y organización, análisis. }\end{array}$ \\
\hline $\begin{array}{l}\text { Planear, gestionar y revisar las } \\
\text { adquisiciones. }\end{array}$ & 27 & $\begin{array}{l}\text { Resolución de problemas, negociación, planeación y organización, } \\
\text { Conocimiento práctico y cálculo, habilidades tecnológicas. }\end{array}$ \\
\hline $\begin{array}{l}\text { Planear, gestionar y revisar la } \\
\text { integración. }\end{array}$ & 31 & $\begin{array}{l}\text { Conocimiento práctico, análisis, trabajo en equipo, comunicación, } \\
\text { manejo del tiempo, planeación y organización. }\end{array}$ \\
\hline
\end{tabular}

\section{Fuente. Elaboración propia.}




\subsection{Investigaciones sobre habilidades y competencias}

En los diferentes artículos consultados, se halló que en el pasado se daba relevancia específicamente a los conocimientos y a las habilidades técnicas, mientras que en la actualidad se reconoce alta importancia a las conocidas como habilidades blandas.

Tett, Guterman, Bleier y Murphy (2000) clasificaron 53 competencias gerenciales en nueve categorías generales: funciones tradicionales, orientación a la tarea, orientación a la persona, mentalidad abierta, control emocional, comunicación, desarrollo propio y de otros, perspicacia, y experiencia ocupacional y organización personal.

El-Sabaa (2001) clasifica las habilidades de un gerente de proyectos en tres categorías: i) habilidades humanas; ii) habilidades conceptuales y organizacionales; y iii) habilidades técnicas. Las primeras se evidencian en la forma como el gerente trabaja en equipo y reconoce las habilidades de superiores, compañeros y subordinados, lo cual le permite motivarlos para alcanzar los objetivos propuestos en su proyecto. Entre estas, se incluyen comunicación, delegación de autoridad, autoestima y entusiasmo, y representan aquellas con mayor relevancia para el desarrollo del gerente. Las habilidades conceptuales y organizacionales permiten al gerente conectarse con la organización y los objetivos de esta, de forma tal que pueda contribuir a su crecimiento viendo el proyecto que lidera como un todo y como parte del conjunto de iniciativas de la organización. En esta categoría, se incluyen habilidades como planeación, organización y orientación a objetivos. Las habilidades técnicas, por otro lado, son aquellas compuestas por metodologías, procesos y procedimientos que aumenten la efectividad con la que el gerente dirige un proyecto, así como conocimientos especializados en el área específica de este. Contrario a lo cual se podría pensar, son estas habilidades las que obtienen menor calificación en cuanto a esencialidad para el perfil gerencial.

En el contexto educativo, Pant y Baroudi (2008) encontraron que son las habilidades duras las que tienen mayor presencia, pues suelen ser más sencillas de enseñar, mientras que las habilidades blandas, ligadas a la inteligencia emocional, suelen ser tratadas de forma más superficial. Es necesario, entonces, equilibrar estos componentes de la enseñanza de la gerencia de proyectos de forma tal que los profesionales logren adquirir todas las habilidades necesarias para una gerencia exitosa.

\subsection{La mujer en la gerencia de proyectos}

En el ámbito internacional, países como los Estados Unidos, Canadá, Nueva Zelanda y Taiwán han comenzado a mostrar un mayor balance en el número de mujeres gerentes de proyectos; sin embargo, otros países, especialmente en América Latina donde la gerencia de proyectos se perfila aún como una disciplina reciente, esta diferencia es bastante grande y tan solo cerca de $10 \%$ de los gerentes de proyectos son mujeres (Burba, 2010). Aun así, la participación de la mujer en la gerencia de proyectos y las diferencias en la cultura de las organizaciones y en las condiciones laborales para ellas, comparadas con las de sus pares hombres, son aspectos que hasta ahora no cuentan con mucho estudio ni documentación, y a menudo son tratados como "un caso especial» (Botkova, 2008), o de forma general para los negocios, sin detallar la situación específica de la gerencia de proyectos. 
De acuerdo con el estudio realizado por Botkova (2008), la división de género en la gerencia de proyectos se mantenía igual en comparación con 2007, con una participación de los hombres de $72 \%$ en contraste con $28 \%$ de mujeres. Los hombres siguen siendo la fuerza dominante, principalmente en los temas de gerencia de proyectos aeroespaciales, en el sector de construcción, consultoría, telecomunicaciones e informática y en la ingeniería en general. Las mujeres participan más en temas relacionados con servicios financieros, salud, seguros, farmacéutica y telecomunicaciones (Klein, 2005, citado por Botkova, 2008). El reporte "Internacional de negocios" -International Business Report-, de Grant Thornton (2014), mostró que solo 66 \% de las empresas del Reino Unido ahora tienen al menos una mujer en un rol de nivel superior o gerencial, en comparación con lo demostrado en la investigación The Official Board 2013, en la que se muestra que China tiene la mayor representación de mujeres como Chief Executive Officer (CEO) -17\%-, mientras que los Estados Unidos muestra apenas 6 \% (McCarthy y Burn, 2013). La más reciente encuesta de salarios realizada por el PMI (2013b), contestada de manera voluntaria por gerentes de proyectos de diferentes países, en Colombia por 332 hombres y 98 mujeres, indica que no solo hay predominio en la participación de los hombres, sino también existen diferencias en la remuneración. En los resultados, se observa, que dentro de los países considerados, en los europeos la brecha salarial es notablemente menor que la que se ve en los dos de América Latina.

En un estudio exploratorio realizado por Henderson y Stackman (2010) se analizó la importancia del género con relación al rol, con la localización del equipo, el uso de tecnología y el costo del proyecto, tras lo cual se encontró que las mujeres como gerentes y como miembros del equipo suelen ser asignadas a proyectos pequeños y de bajo costo, marginándolas de la visibilidad que puede dar el estar a cargo de un proyecto de alta importancia para sus organizaciones.

En un estudio realizado para el sector de las tecnologías de información (Legault y Chasserio, 2012), encontraron que una de las principales razones por las cuales se limita la participación de las mujeres como gerentes de proyectos es su necesidad de tiempo para dedicar a la familia y a ellas mismas, lo cual reduce el tiempo de su jornada laboral considerado como uno de los factores que indica compromiso con la organización y sus proyectos.

Arditi y Balci (2009) muestran que las mujeres son tan competentes como los hombres en el desempeño como gerentes de proyectos en el sector de la construcción. Las calificaciones de las mujeres fueron superiores en 17 de las competencias evaluadas, sin embargo, solo en tres de ellas las diferencias fueron estadísticamente significativas: sensibilidad, enfoque en el cliente y autoridad y presencia.

De acuerdo con Kearney y Lloyd (2007), más de la mitad de las mujeres en el Reino Unido $-54 \%$ - manifiesta haber tenido algún tipo de discriminación en la selección, contratación o durante el desarrollo de su trabajo. En la investigación, se entrevistó a 2256 personas -1047 hombres y 1209 mujeres - de diversas edades, profesiones y antigüedad en el Reino Unido. Casi cuatro de cada diez mujeres -39 \%- afirmaron que el sector en el que trabajan es dominado por hombres. 


\section{Metodología}

Dl desarrollo de esta investigación se Easa en la realización de un estudio cualitativo a través de la aplicación de un cuestionario estructurado en línea a mujeres que participan en roles de gerencia de proyectos utilizando la herramienta de formularios de Google. Con el fin de asegurar la participación de mujeres gerentes de proyectos, dentro del cuestionario se verifica si quien responde es mujer o es hombre. En este mismo sentido, se verificaron los datos personales de quien respondía, los cuales se recopilaban al final del cuestionario en la sección información general. A continuación, se presenta la metodología aplicada en cuanto a enfoque, limitaciones, búsqueda de la información y preparación del instrumento aplicado.

En lo referente al enfoque de la investigación, dada la naturaleza del estudio que se adelanta, se consideró apropiado seleccionar un enfoque cualitativo, puesto que se pretende comprender algunas características propias de un conjunto de una población, mediante los datos obtenidos a través de una aproximación subjetiva, debido a que se basa en las percepciones y experiencias de quienes participen en el estudio. Esta investigación se encuentra limitada por aspectos como el tiempo de ejecución y acceso a los participantes. Se encuentra limitado al entorno colombiano, particularmente a mujeres gerentes de proyectos que pudieron ser contactadas a través de redes sociales y el capítulo PMI Colombia, lo cual podría limitar las conclusiones respecto de regiones y sectores. Esta investigación no incluye hombres gerentes de proyectos, sin embargo, en la sección recomendaciones y trabajo futuro se incluye este aspecto para futuras investigaciones.
En cuanto a la búsqueda de información, se realizaron búsquedas de palabras clave relacionadas con el tema objeto de la investigación, en las bases de datos EBSCO, Science Direct y en los buscadores Google Scholar y Google, así como páginas web específicas como la del PMI (www.pmi.org), DANE (www.dane.gov.co) y el PNUD (www.pnud. org.co). Se tomaron como criterios de selección para los resultados encontrados que se tratara de artículos, libros o publicaciones realizadas en los últimos diez años.

Entre los diferentes tipos de instrumentos, para esta investigación se determinó la realización de una encuesta estructurada, debido, en primer lugar, al corto tiempo disponible para su aplicación, la posibilidad de aplicarla en línea y tener acceso a una mayor cantidad de respuestas; de esta manera lograr establecer contacto con mujeres que se desempeñen en la gerencia de proyectos, idóneas para participar en este estudio y en fases posteriores del proyecto de investigación.

Teniendo como base la revisión de literatura realizada, se planteó un listado inicial de los aspectos por indagar para determinar el rol de la mujer en la gerencia de proyectos, los cuales fueron discutidos en cinco reuniones con los profesores del Centro de Estudios en Desarrollo y Gerencia Integral de Proyectos de la Escuela Colombiana de Ingeniería Julio Garavito, quienes oficiaron como expertos, uno de ellos en aspectos metodológicos. Allí se analizaron los temas considerados pertinentes, y se determinaron y estructuraron una a una las preguntas que formarían parte de la encuesta. En su versión definitiva, la encuesta contiene 55 preguntas generales, para ejemplificar, 
estructurales y de contraste, clasificadas en cinco diferentes categorías:

- Características generales, con referencia a la situación específica de cada persona.

- Trayectoria profesional sobre el recorrido en su experiencia laboral.

- Rol de la mujer como gerente de proyectos en la organización considerando aspectos relativos al género en el ejercicio de sus funciones.

- Desempeño de la mujer en la gerencia de proyectos sobre aspectos de la gestión propia y su percepción frente a pares hombres.
- Información general y preguntas de respuesta opcional para identificación y datos de contacto.

El formulario fue difundido a través de las redes sociales Facebook, Twitter y LinkedIn, y enviado por correo electrónico tanto en forma abierta como dirigida a grupos de interés específicos, tales como el Capítulo Colombia del PMI y los graduados y estudiantes actuales de la especialización y maestría en Desarrollo y Gerencia Integral de Proyectos de la Escuela Colombiana de Ingeniería Julio Garavito, entre otros. En todos los casos, se solicitó, a quienes vieran la información, que la compartieran con sus contactos. Se logró la participación de 21 personas (Anexo 1).

\section{Resultados}

\subsection{Características generales}

De acuerdo con los resultados de la encuesta, los sectores de consultoría y de tecnologías de la información presentan las mayores participaciones $-24 \%$ cada uno - , seguidos del sector de petróleo y gas y de la construcción - $14 \%$ cada uno-.

En cuanto al entorno familiar, los resultados muestran que las gerentes de proyectos objeto de este estudio son mujeres principalmente entre los 25 y los 40 años -61 \%-. El $48 \%$ de los resultados corresponde a mujeres casadas, de las cuales la mayoría es mayor de 33 años $-80 \%$-. En cuanto al número de hijos, el promedio es de menos de uno por familia, presentando un porcentaje importante $-47 \%$ - de mujeres sin hijos.

El nivel educativo es predominantemente de posgrado, siendo la especialización -52 $\%$ - el grado más destacado. Esta preferencia se mantiene específicamente para el nivel de educación en gerencia de proyectos, aunque en un porcentaje menor $-38 \%-$. Cabe destacar que en esta última característica se presenta un porcentaje importante -24 $\%$ - de mujeres sin formación específica en gerencia de proyectos. 


\subsection{Trayectoria profesional}

De acuerdo con los resultados de la encuesta, la trayectoria profesional en gerencia de proyectos de la población de mujeres objeto de este estudio es relativamente corta en comparación con su vida laboral. Mientras que más de $50 \%$ de las mujeres respondió tener más de diez años de experiencia laboral,
$67 \%$ de ellas aseguró tener menos de cinco años de experiencia específica en gerencia de proyectos. Esta condición se corrobora al analizar la cantidad de años requeridos para alcanzar el rol de gerente de proyecto (Figura 1). Sin embargo, esto también muestra un interés reciente de las mujeres por incursionar en el ámbito de la gerencia de proyectos como parte de su perfil profesional.

Figura 1. Años de experiencia transcurridos desde el título profesional hasta ocupar el rol de gerente de proyectos.

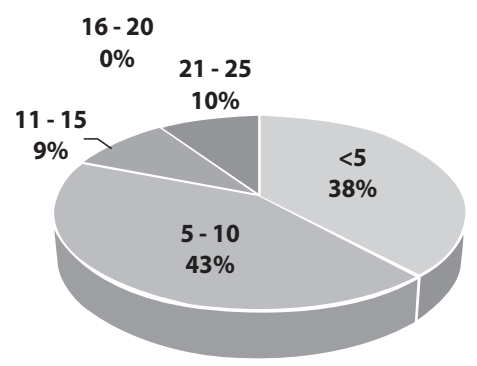

Fuente. Elaboración propia.

Otro aspecto sobre el que se indagó en la encuesta fue acerca de las habilidades que las mujeres consideran importantes dentro de su perfil para el desarrollo de la gerencia de proyectos. Los resultados muestran que el liderazgo, la capacidad de toma de decisiones y las habilidades de comunicación son las más sobresalientes dentro de sus perfiles (Figura 2). Este resultado concuerda plenamente con lo encontrado en la literatura en cuanto a las habilidades fundamentales para un gerente de proyectos donde el liderazgo y la comunicación son algunas de las que se encuentran de forma más recurrente en los diferentes modelos analizados en el marco teórico.

Figura 2. Habilidades gerenciales importantes en el perfil propio.

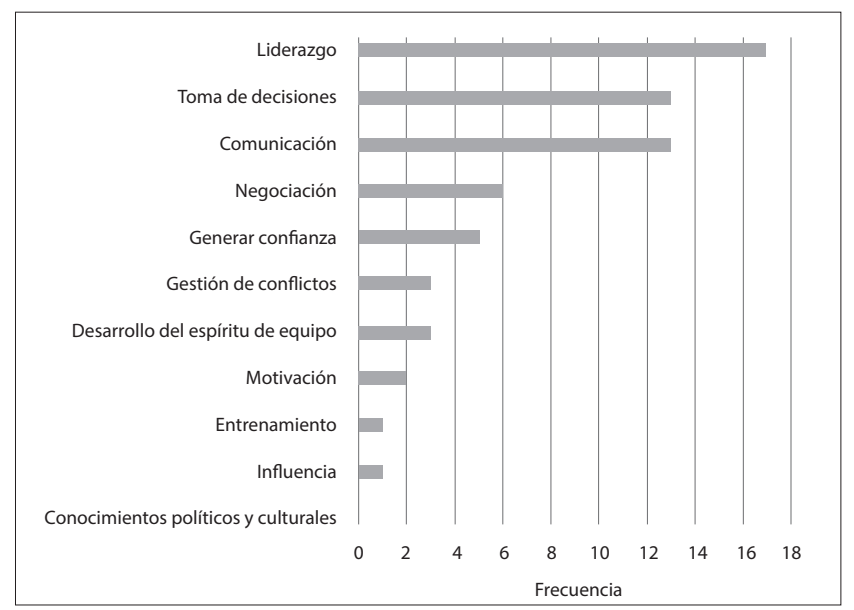

Fuente. Elaboración propia. 
Según la encuesta, las mujeres, en su mayoría $-62 \%-$, tienen suficiente autoridad para la toma de decisiones respecto del desarrollo de sus proyectos; sin embargo, una cantidad considerable $-33 \%$ - señala tener ciertas restricciones para la toma de decisiones. Solo una pequeña parte $-5 \%-$ debe consultar la mayoría de sus decisiones.

Con respecto a la percepción que tienen las mujeres encuestadas sobre la facilidad de acceso al mercado laboral y la dificultad para alcanzar posiciones gerenciales en proyectos, los resultados hacen ver que ellas sienten una clara preferencia del mercado por sus pares hombres. Las respuestas a las razones de esta apreciación se relacionan, de acuerdo con lo mencionado por las mujeres encuestadas en comentarios complementarios, con la creencia generalizada de que los hombres poseen mejores capacidades de liderazgo y control de recursos, así como el riesgo que se percibe desde empresas contratantes en cuanto a limitaciones en la disponibilidad de tiempo de la mujer debido a roles familiares. Una vez dentro de la organización, el tiempo que toma a hombres y mujeres para llegar a ocupar las posiciones de gerencia de proyectos es igual; menor en algunos casos para los hombres, pero no se percibe que este tiempo pueda ser menor para las mujeres.

Finalmente, se preguntó a las mujeres sobre la influencia que ejerce su entorno familiar en su trayectoria y en su desempeño como gerentes de proyectos. Las respuestas a esta pregunta sugieren que este aspecto de la vida de las mujeres ejerce una fuerte influencia en su trayectoria $-66 \%-$ y en un porcentaje importante $-43 \%$ - también en su desempeño. Cabe aclarar que esta influencia puede ser positiva, impulsándolas a mejorar cada día; o negativa, limitando su crecimiento profesional en favor del desarrollo de su vida familiar.

\subsection{Rol de la mujer gerente de proyectos en la organización}

Con referencia al porcentaje de mujeres que desempeñan roles de gerencia de proyectos en la organización, la encuesta mostró que esta participación se mantiene baja y en su mayoría se encuentra por debajo de $50 \%$. Este resultado puede estar directamente relacionado con lo mencionado en la categoría anterior en cuanto a preferencia del mercado por contratar hombres para los roles gerenciales y con el hecho de que a ellas les tome más tiempo llegar a estas posiciones. Sin embargo, de acuerdo con las respuestas de la encuesta, estas preferencias de género no se hacen de manera explícita en las convocatorias de trabajo.

La compensación salarial es otro punto que aún preocupa a las mujeres en el ejercicio laboral, no solo en la gerencia de proyectos. De acuerdo con los resultados, las mujeres encuestadas, en su mayoría, sienten igualdad de condiciones salariales con sus pares hombres. Sin embargo, un porcentaje aún importante $-33 \%$ - cree que esta situación no es favorable a las mujeres, y en ningún caso se presenta la percepción de tener una remuneración mayor ligada a la condición de género.

Una vez dentro de la organización, los resultados de la encuesta muestran que no se perciben grandes diferencias entre hombres y mujeres en cuanto a funciones, responsabilidad y asignación de recursos. Sin embargo, en el poder y los niveles de autoridad, un porcentaje mayor de mujeres manifiesta percibir diferencias frente a los hombres (Figura 3 ). Esto se debe, de acuerdo con las respuestas de las encuestadas, a una mayor participación de hombres en los niveles de liderazgo y por tanto un mayor poder de decisión que en ocasiones incluye la validación de las decisiones tomadas por las mujeres. 
Figura 3. Identificación de diferencias entre mujeres y hombres gerentes de proyectos en cuanto a funciones, responsabilidades, niveles de autoridad, asignación de recursos y poder.
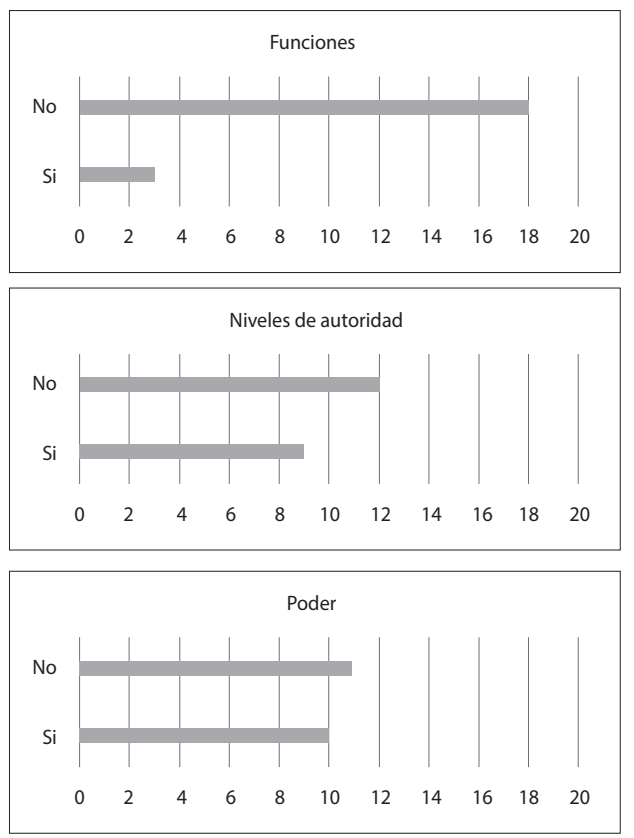

Fuente. Elaboración propia.

Las organizaciones a las que pertenecen las mujeres encuestadas les otorgan poder para la toma de decisiones mediante su designación en cargos de responsabilidad y mediante la posibilidad de elegir su propio equipo de trabajo. Además, se muestra el interés de algunas de las organizaciones por fomentar el liderazgo en las mujeres, aunque este aspecto aún tiene espacio para mejorar, pues el porcentaje de las organizaciones en las que no se tiene este tipo de políticas es considerable $-38 \%$ -

De acuerdo con las respuestas de la encuesta, las mujeres, en su mayoría, no tienen preferencia en cuanto al género de su superior inmediato; sin embargo, es de resaltar, que de aquellas que prefieren un género específico, el porcentaje más bajo es para la preferencia de jefe mujer, dado que según comentarios de las encuestadas, con un jefe hombre se reducen la competencia

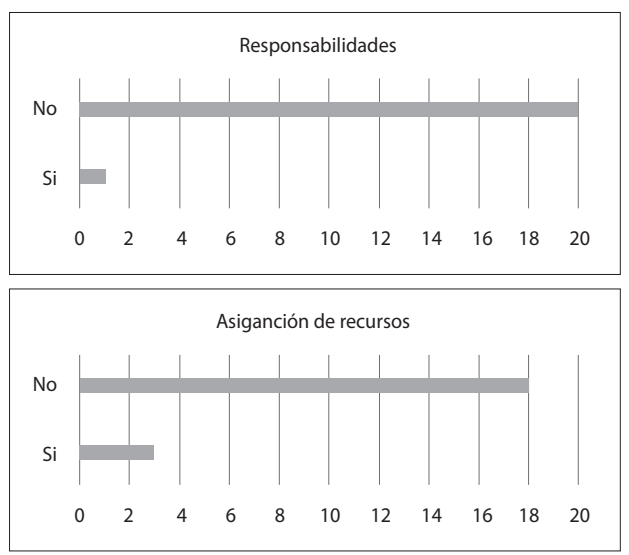

de género y el aspecto emocional que se percibe podría afectar su objetividad. Además, se señala que las mujeres suelen ser más detallistas y exigentes respecto de los resultados que esperan de sus equipos de trabajo, lo cual en ocasiones genera roces con sus integrantes.

\subsection{Desempeño de la mujer en la gerencia de proyectos}

Según los resultados de la encuesta, la mayoría de los proyectos liderados por las mujeres encuestadas han obtenido resultados positivos en cuanto a objetivos y presupuesto, en una frecuencia superior a $70 \%$ de las veces en que las mujeres han tenido esta responsabilidad. Sin embargo, se ve una tendencia a disminuir el cumplimiento del cronograma, aunque esta frecuencia aún se encuentra por encima de $50 \%$.

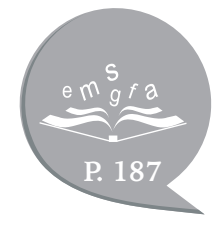


Al considerar de forma general el éxito de los proyectos, la encuesta muestra que las mujeres, de acuerdo con los criterios internos de las organizaciones, consideran exitosos la mayoría de los proyectos liderados por ellas y que no perciben diferencia en el desempeño de aquellos liderados por sus pares hombres; por el contrario, los gerentes de proyectos, independiente de su género, pueden alcanzar resultados positivos dependiendo de condiciones diferentes de la de género.

Por otro lado, se muestra que las mujeres identifican que superan a los hombres en sus habilidades aplicadas a la gerencia de proyectos en lo concerniente a la generación de confianza, comunicación, motivación de los equipos de proyecto y negociación (Figura 4).

A partir de las respuestas de las mujeres encuestadas, en las organizaciones a las que ellas pertenecen, claramente existen políticas de equidad de género en cuanto a remuneración, estímulos, educación, capacitación, representación en cargos directivos, desarrollo de carrera y contratación, y en la mayoría de los casos estas políticas se aplican total o parcialmente. Así mismo, se incentiva la participación activa de la mujer en la gerencia de proyectos.

Figura 4. Habilidades más desarrolladas por mujeres que por hombres.

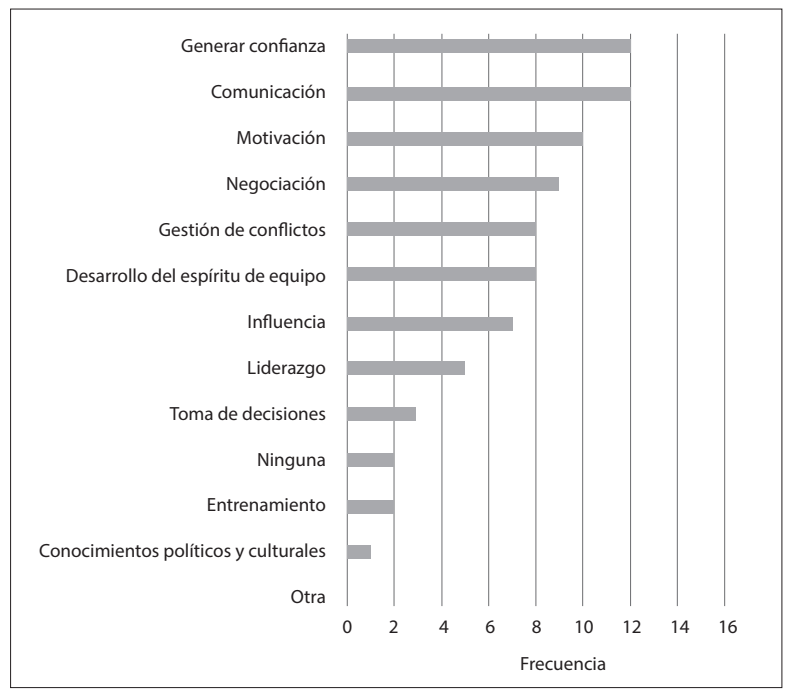

Fuente. Elaboración propia.

Otro aspecto importante indagado en la encuesta tiene que ver con el reconocimiento de diferencias entre hombres y mujeres en aspectos como la forma de afrontar los problemas, definición de estrategias, actitud frente al trabajo en equipo, personalidad y actitud ante los riesgos. Los resultados muestran que en su mayoría no se perciben diferencias en estos aspectos entre los géneros, y específicamente en personalidad se nota un mayor equilibrio entre las percepciones. De acuerdo con las respuestas de las mujeres encuestadas, las organizaciones y los equipos en general no tienen interiorizadas las diferencias inherentes entre los géneros, pero se perciben implícitas en las actitudes de los equipos. 
Con respecto al futuro de su carrera profesional, $57 \%$ de las mujeres encuestadas manifestó que las organizaciones a las cuales pertenecen presentan para ellas iguales oportunidades de ascender dentro de la empresa y se nota un claro compromiso de la alta dirección por apoyar a la mujer desarrollando planes de carrera que les permitan crecer dentro de la organización.

La complejidad y los costos de los proyectos, según la experiencia de las mujeres encuestadas, tampoco presentan grandes diferencias entre hombres y mujeres gerentes de proyectos. Sin embargo, es de resaltar que $28 \%$ de las mujeres encuestadas percibe que los proyectos asignados a ellas suelen presentar un menor nivel de complejidad y presupuestos más bajos que aquellos asignados a su contraparte masculina.

Finalmente, se realizaron dos preguntas específicas sobre discriminación a la mujer tanto en la organización como en su vida profesional en general. De acuerdo con estos resultados, más de $80 \%$ de las mujeres encuestadas asegura no sentir discriminación en la organización en la que labora actualmente. Sin embargo, más de $50 \%$ de ellas asegura haber sufrido discriminación en algún momento de sus carreras, por lo cual es posible pensar que esta condición ha venido mejorando en los últimos tiempos brindándoles un ambiente de trabajo más propicio para su desarrollo como profesionales en la gerencia de proyectos.

\section{Conclusiones}

\begin{abstract}
A continuación, se presentan las concluAsiones del estudio realizado, organizadas de acuerdo con la estructura de la encuesta aplicada y finalizando con el perfil de la mujer gerente de proyectos a partir de las respuestas obtenidas.
\end{abstract}

\subsection{Características generales}

Una alta proporción de las gerentes de proyectos encuestadas cuenta con título de posgrado, $52 \%$ con especialización, $24 \%$ con maestría y $5 \%$ con doctorado. Es posible que este comportamiento de los resultados esté relacionado con el hecho de que algunos de los principales canales utilizados para compartir el formulario, correo electrónico y Facebook ofrecen contacto directo con los graduados de posgrado de la Escuela Colombiana de Ingeniería Julio Garavito, lo cual puede corroborarse con la cantidad de mujeres con especialización en el área de proyectos, específicamente $-38 \%$ - Sin embargo, $24 \%$ no cuenta con formación específica en el tema.

Las mujeres que respondieron la encuesta muestran, de acuerdo con los resultados, que han desarrollado su vida familiar en condiciones relativamente normales, a pesar de sus compromisos por el rol gerencial, pues $48 \%$ de ellas están casadas y tienen hijos, y las solteras $-43 \%$ - se encuentran en los rangos de edad inferiores, menores de 33 años. 
En lo relacionado con los ingresos anuales de las mujeres gerentes de proyectos, comparando los resultados de la encuesta con los presentados como resultado de la Salary Survey 2013 del PMI ( 2013b), los ingresos de $57 \%$ de las mujeres encuestadas en este estudio se encuentran en los rangos inferiores al que incluye el valor promedio -COP 89.430.102-, correspondiente a los ingresos delas 98 mujeres que participaron en la encuesta del PMI. Por otro lado, mientras que en esta última se aprecia que el promedio de los ingresos de los hombres es mayor que el de las mujeres, $33 \%$ de las mujeres que participaron en este estudio percibe, que en general, la compensación recibida por los hombres es mayor a la recibida por las mujeres, mientras que ninguna de ellas percibe la situación contraria.

\subsection{Trayectoria profesional}

Las habilidades consideradas como las más importantes en su perfil por las gerentes de proyectos encuestadas son en orden de importancia: el liderazgo, la toma de decisiones, de comunicación, de negociación y generación de confianza en el equipo. Las habilidades mencionadas forman parte de aquellas propuestas en los estándares internacionales, específicamente el del PMI, en el anexo X3 de la Guía del PMBOK ${ }^{\circledR}$ (PMI, 2013a).

Las mujeres gerentes de proyectos que respondieron la encuesta ostentan autoridad: $62 \%$ de ellas tiene autoridad alta o total, lo cual les permite tomar decisiones y desempeñar su rol sin limitaciones significativas. Sin embargo, detectan algunas restricciones para ingresar al mercado laboral y para ascender a cargos gerenciales, debido a la idea de que existe para ellas una gran necesidad de tiempo para dedicar a sus familias. De la misma forma que determinaron Legault y Chasserio (2012) en su estudio realizado para el sector de tecnologías de información, según el cual esta condición reduce el tiempo de su jornada laboral, y por tanto, de compromiso con la organización y sus proyectos. Esta podría ser una de las principales razones por las cuales algunas mujeres sienten influencia de su entorno familiar en su trayectoria profesional y en su desempeño como gerente de proyectos. Además, algunos de sus comentarios se refieren a dificultades para acceder a cargos en gerencia de proyectos debido al predomino y la tradición del género masculino en estos roles y a la creencia de que los hombres poseen mejores habilidades para el liderazgo.

\subsection{Rol de la mujer gerente de proyectos en la organización}

Mientras que no se perciben diferencias significativas en la asignación de funciones y responsabilidades entre gerentes de proyectos hombres y mujeres, sí hay percepción de que a los hombres gerentes de proyectos se les otorga mayor poder debido a que se les asignan proyectos de mayor costo y alcance. Esto concuerda con lo expuesto por Henderson y Stackman (2010) en los resultados de su estudio.

Se aprecia, que en general, se cuenta con un ambiente propicio para que las mujeres desarrollen su carrera como gerentes de proyectos, dado que las respuestas a las preguntas relacionadas con el apoyo de las organizaciones y las oportunidades que estas ofrecen particularmente al género femenino son positivas. Sin embargo, ellas perciben que existen preferencias para la contratación de hombres, y a lo largo de su carrera han sido víctimas de discriminación por género. 
A pesar de lo anterior, la participación de las mujeres en roles de gerencia de proyectos en organizaciones sigue siendo relativamente baja, $52 \%$ responde que en sus organizaciones menos de $25 \%$ de los gerentes de proyectos son mujeres.

\subsection{Desempeño de la mujer en la gerencia de proyectos}

Las mujeres encuestadas consideran que, en las habilidades de generación de confianza en el equipo, comunicación, motivación y negociación, cuentan con mayor desarrollo cual sus pares hombres. Esto contrasta con los resultados del estudio realizado (Arditi y Balci, 2009), según los cuales hombres y mujeres cuentan con niveles similares de competencias para las labores de gerencia de proyectos.

De acuerdo con los resultados de este trabajo, las mujeres encuestadas consideran tener las capacidades para conducir exitosamente el desarrollo de un proyecto, ya que, en general, la mayoría de ellas -62 \%manifiesta que ha cumplido con los resultados en alcance y costo en más de $75 \%$ de los proyectos que han dirigido. En cuanto al cumplimiento de los objetivos en tiempo, este parece presentar el mayor espacio de mejora, pues solo $42 \%$ de las mujeres encuestadas aseguró cumplir este factor en más de $75 \%$ de los proyectos.

En cuanto a reconocimiento de diferencias entre hombres y mujeres por parte de la organización, una de las encuestadas manifiesta: «A las mujeres se le delegan responsabilidades relacionadas con el control y cumplimiento de requisitos y a los hombres con gestión y cumplimiento de objetivosı, lo cual concuerda con las principales funciones que mencionan las encuestadas como parte de su labor.
Por otro lado, se aprecia la existencia de políticas de equidad de género en las organizaciones en las que trabajan las mujeres encuestadas, situación que concuerda con lo requerido en los artículos 13 y 43 de la Constitución Política, el artículo 143 del Código Sustantivo del Trabajo, y la Ley 1496 de 2011. Sin embargo, se aprecia un espacio importante para continuar mejorando este aspecto en las organizaciones, puesto que de acuerdo con los resultados de este estudio y la percepción de las mujeres encuestadas, las políticas existen y se aplican totalmente en menos de la mitad de los casos, especialmente en los aspectos de desarrollo de carrera y representatividad en cargos directivos.

Finalmente, aunque las mujeres que participaron en el estudio manifestaron que en general no han sido víctimas de discriminación en las organizaciones en que trabajan, sí la han sentido en el ejercicio de la gerencia de proyectos, lo cual concuerda con los resultados del estudio realizado por Kearney y Lloyd (2007), según el cual cerca de la mitad de las mujeres se han sentido víctimas de discriminación en la selección, la contratación o el ejercicio de sus funciones como gerentes de proyectos.

\subsection{El perfil de la mujer gerente de proyectos}

De acuerdo con los resultados, el perfil general de las mujeres que respondieron a la encuesta es el de profesionales con posgrado, con formación específica en gerencia de proyectos, en su mayoría en especialización, de más de 27 años, con habilidades de liderazgo, toma de decisiones y comunicación, seguidas de capacidad de negociación y de generar confianza en el equipo; además, perciben que superan a los gerentes de proyectos hombres en sus habilidades para generar confianza, comunicación y motivación (Tabla 4). 
Tabla 4. Perfil de la mujer gerente de proyectos

\begin{tabular}{|c|c|c|}
\hline Sector de la organización & $\begin{array}{l}\text { Tecnologías de la información } \\
\text { Consultoría } \\
\text { Construcción } \\
\text { Petróleo y gas } \\
\text { Otros }\end{array}$ & $\begin{array}{l}25.0 \% \\
20.0 \% \\
15.0 \% \\
15.0 \% \\
25.0 \%\end{array}$ \\
\hline Nivel educativo & $\begin{array}{l}\text { Especialización } \\
\text { Maestría } \\
\text { Pregrado } \\
\text { Doctorado } \\
\text { Tecnólogo }\end{array}$ & $\begin{array}{c}52.4 \% \\
23.8 \% \\
14.3 \% \\
4.8 \% \\
4.8 \%\end{array}$ \\
\hline Nivel educativo en gerencia de proyectos & $\begin{array}{l}\text { Especialización } \\
\text { Sin formación específica } \\
\text { Diplomado } \\
\text { Maestría } \\
\text { Curso }\end{array}$ & $\begin{array}{c}38.1 \% \\
23.8 \% \\
19.0 \% \\
9.5 \% \\
9.5 \%\end{array}$ \\
\hline Años de experiencia general & $\begin{array}{l}5-10 \\
16-20 \\
21-25 \\
\text { Menos de } 5 \\
26-30 \\
11-15\end{array}$ & $\begin{array}{l}33.0 \% \\
19.0 \% \\
14.0 \% \\
14.0 \% \\
10.0 \% \\
10.0 \%\end{array}$ \\
\hline Años de experiencia específica en gerencia de proyectos & $\begin{array}{l}\text { Menos de } 5 \\
16-20 \\
5-10 \\
11-15 \\
21-25\end{array}$ & $\begin{array}{c}67.0 \% \\
10.0 \% \\
9.0 \% \\
9.0 \% \\
5.0 \%\end{array}$ \\
\hline Tres habilidades más importantes & $\begin{array}{l}\text { Liderazgo } \\
\text { Toma de decisiones } \\
\text { Comunicación } \\
\text { Negociación } \\
\text { Generación de confianza } \\
\text { Gestión de conflictos } \\
\text { Desarrollo del espíritu de equipo } \\
\text { Motivación } \\
\text { Entrenamiento } \\
\text { Influencia }\end{array}$ & $\begin{array}{l}81.0 \% \\
61.9 \% \\
61.9 \% \\
28.6 \% \\
23.8 \% \\
14.3 \% \\
14.3 \% \\
9.5 \% \\
4.8 \% \\
4.8 \%\end{array}$ \\
\hline
\end{tabular}

\section{Fuente. Elaboración propia.}




\section{Recomendaciones y trabajo futuro}

E primer lugar, considerando los resultados obtenidos en la investigación realizada en este trabajo, que corresponde a la parte inicial de un proyecto de investigación a cargo del Centro de Estudios en Desarrollo y Gerencia Integral de Proyectos, adscrito a la unidad de proyectos de la Escuela Colombiana de Ingeniería Julio Garavito, y la relevancia del tema para el desarrollo de la gerencia de proyectos como profesión, se recomienda extender el alcance de la investigación a un mayor número de mujeres que se desempeñen en el ámbito de la gerencia de proyectos, de manera que se cuente con suficiente cantidad de información para analizar posibles diferencias de perfiles en distintos sectores y regiones, y realizar ajustes tanto al cuestionario como a algunos aspectos metodológicos de la realización de la encuesta. Además, para poder encontrar aspectos específicos del perfil de la mujer como gerente de proyectos considerando particularmente la condición de género, es conveniente incluir en la investigación a hombres o hacer una investigación específica sobre el perfil del hombre gerente de proyectos, con lo cual podrán evidenciarse posibles similitudes y diferencias entre sus perfiles.

\subsection{Ajustes al cuestionario}

En la primera pregunta del cuestionario, se pretende obtener información sobre el sector en que se desempeña la encuestada, de tal forma que se logre una segmentación por tipo de industria. La lista fue tomada inicialmente del cuestionario de la encuesta que realiza el PMI (2013b); sin embargo, durante el análisis de los resultados de la encuesta se pudo apreciar que algunos de los elementos pueden generar confusiones y respuestas ambiguas. Por ejemplo, una organización de ingeniería o consultoría podría realizar trabajos de construcción, tecnologías de la información o telecomunicaciones, entre otras. En cuanto a la educación, sería conveniente conocer, al menos, el área o la carrera de formación en pregrado y el programa y la institución específica en gerencia de proyectos.

La pregunta en que se indaga sobre las funciones y responsabilidades como gerente de proyectos es abierta; las respuestas obtenidas en la encuesta resultaron poco específicas, por lo cual no permiten realizar suficiente análisis. Se recomienda modificarla para que sea de tipo cerrada, de selección múltiple con múltiple respuesta.

La influencia del entorno familiar en la trayectoria o el desempeño de una gerente de proyectos podría ser positiva o negativa; solo se pregunta si existe tal influencia, por lo cual sería conveniente complementar la pregunta con el tipo de influencia. En la última pregunta del cuestionario, sobre la discriminación que hayan podido sufrir las mujeres en el ejercicio de la gerencia de proyectos, se sugiere ahondar acerca de la forma en que esta se ha experimentado.

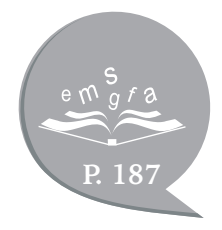




\section{Referencias}

Arditi, D. y Balci, G. (2009). Managerial competencies of female and male construction managers. Journal of Construction Engineering and Management, 135(11), 1275-1278.

Australian Institute of Project Management (2010). AIPM Professional Competency Standards for Project Management Part C-Certified Practising Project Manager (CPPM). Sídney: The Australian Institute of Project Management.

Australian Institute of Project Management (2015). About us. Recuperado de https://www.aipm.com. au/about-us

Botkova, Z. (2008). The Progression of Women in Project Management. Middlesex University.

Burba, D. (2010). Equal ground? They earn less. They're stereotyped. They have to fight for respect. Are women faring any better in project management? PM Network, 24(9), 38-42.

Crowe, A. (2008). Alpha Project Managers. Kennesaw: Velociteach Press.

El-Sabaa, S. (2001). The skills and career path of an effective project manager. International Journal of Project Management, 19, 1-7.

Giraldo, G. E., Pulido, G. H. y Leal, C. A. (2013). Project Manager Profile Characterization in the Construction Sector in Bogotá, Colombia. Project Management Journal, 44(6), 68-93.

Thornton, G. (2014). Women in business: From classroom to boardroom Grant Thornton International Business Report 2014. Grant Thornton.

Harvard Law School (2015). PON-Program on Negotiation at Harvard Law School. Recuperado de http://www.pon.harvard.edu/category/daily/ negotiation-skills-daily/

Henderson, L. S. y Stackman, R. W. (2010). An exploratory study of gender in project management: Interrelationships with role, location, technology, and project cost. Project Management Journal, 41(5), 37-55.
International Project Management Association (2006). ICB-IPMA Competence Baseline Version 3.0 (3. ${ }^{\mathrm{a}}$ ed.). Nijkerk, The Netherlands: International Project Management Association.

International Project Management Association (2015). About IPMA. Recuperado de http://ipma. ch/about/

Karch, D. M. (2012). Gender-based comparison of project manager leadership behaviours: A quantitative study (Tesis de doctorado, Capella University, Mineápolis, Minnesota).

Kearney, L. y Lloyd, J. (2007). Half of women encounter discrimination at work. Recuperado de http://www. atkinsglobal.com/en-gb/media-centre/newsreleases/2007/group/half-of-women-encounter

Kerzner, H. y Salidis, F. (2009). Value-driven project management. Hoboken, NJ: John Wiley y Sons.

Klein, K. E. (2005). ProjectsAtWork-It's A Woman's World, Too. Recuperado de https://www. projectmanagement.com/articles/225150/Its-AWomans-World--Too

Legault, M.-J. y Chasserio, S. (2012). Professionalization, risk transfer, and the effect on gender gap in project management. International Journal of Project Management, 30(6), 697-707. Recuperado de http://linkinghub.elsevier.com/ retrieve/pii/S0263786311001505

Levin, G. (2010). Interpersonal skills for portfolio, program, and project managers. Vienna, VA: Management Concepts.

McCarthy, K. y Burn, P. (2013). The Double Glass Ceiling. Recuperado de https://goo.gl/DVUFnW

Neuhauser, C. (2007). Project manager leadership behaviors and frequency of use by female project managers. Project Management Journal, 38 (1), 21-31.

Pant, I. y Baroudi, B. (2008). Project management education: The human skills imperative. International Journal of Project Management, 26, 124128.

PMI (2007). Project Manager Competency Development (PMCD) Framework. 
PMI (2013a). Guía de los fundamentos para la dirección de proyectos. Newtown Square, Pennsylvania: PMI.

PMI (2013b). Project Management Salary Survey Eighth Edition.

Tett, R. P., Guterman, H. A., Bleier, A. y Murphy, P. J. (2000). Development and Content Validation of a "Hyperdimensional" Taxonomy of Managerial Competence. Human Performance, 13, 206-251. 


\section{Anexo 1. Cuestionario aplicado en la investigación}

\begin{tabular}{|c|c|}
\hline \multicolumn{2}{|r|}{ Características generales } \\
\hline 1 & Por favor, seleccione, entre las siguientes categorías, la que más se ajuste al enfoque de su organización. \\
\hline 2 & Nombre de la ciudad donde trabaja (base) \\
\hline 3 & ¿Cuál es la denominación de su cargo? \\
\hline 4 & Edad (años cumplidos) \\
\hline 5 & Género \\
\hline 6 & Nivel de ingresos \\
\hline 7 & Estado civil \\
\hline 8 & Número de hijos \\
\hline 9 & Nivel educativo \\
\hline 10 & Nivel educativo en gerencia de proyectos \\
\hline \multicolumn{2}{|r|}{ Trayectoria profesional } \\
\hline 11 & ¿Cuántos años de experiencia general tiene? \\
\hline 12 & ¿Cuántos años de experiencia específica en gerencia de proyectos ha acumulado? \\
\hline 13 & $\begin{array}{l}\text { ¿Cuántos años transcurrieron desde cuando obtuvo su título profesional hasta cuando llegó a ocupar el cargo de } \\
\text { gerente de proyectos o su equivalente? }\end{array}$ \\
\hline 14 & $\begin{array}{l}\text { Entre las siguientes opciones, ¿cuáles son las tres habilidades gerenciales que considera son las más importantes } \\
\text { en su propio perfil para la gerencia de proyectos? }\end{array}$ \\
\hline 15 & $\begin{array}{l}\text { Seleccione, entre las siguientes opciones, la que mejor describe el grado o nivel de autoridad que actualmente se } \\
\text { le ha otorgado como gerente de proyectos: total, alta, media, baja }\end{array}$ \\
\hline 16 & ¿Cuáles son sus principales funciones y responsabilidades como gerente de proyectos? \\
\hline 17 & $\begin{array}{l}\text { Desde su percepción, ¿se detecta en el mercado laboral preferencia por contratar hombres o mujeres para cargos } \\
\text { en gerencia de proyectos? }\end{array}$ \\
\hline 18 & ¿Por qué? \\
\hline 19 & $\begin{array}{l}\text { Desde su percepción y experiencia considera que en condiciones similares, para que un hombre llegue a ocupar el } \\
\text { cargo de gerente de proyectos o equivalente, le toma más tiempo, menos tiempo, el mismo tiempo }\end{array}$ \\
\hline \multicolumn{2}{|r|}{ Rol de la mujer gerente de proyectos en la organización } \\
\hline 20 & En su organización, el porcentaje de mujeres que se desempeña en el rol de gerentes de proyectos es: \\
\hline 21 & $\begin{array}{l}\text { Desde su perspectiva, ¿cuáles de los siguientes aspectos tienen mayor incidencia en la selección de género de un } \\
\text { gerente de proyectos? }\end{array}$ \\
\hline 22 & $\begin{array}{l}\text { ¿En las convocatorias y procesos de selección para contratar gerentes de proyectos se hace explícito el factor } \\
\text { género? }\end{array}$ \\
\hline 23 & $\begin{array}{l}\text { Según su percepción, la compensación (remuneración, bonificaciones y otros beneficios) recibida por gerentes de } \\
\text { proyectos en la organización en que trabaja, es: }\end{array}$ \\
\hline 24 & $\begin{array}{l}\text { En la organización en que trabaja, ¿existen diferencias entre hombres y mujeres gerentes de proyectos, con } \\
\text { respecto a...? }\end{array}$ \\
\hline 25 & Específicamente, ¿qué diferencias percibe? \\
\hline 26 & Respecto de mi entorno familiar, pienso que... \\
\hline 27 & En la organización en que trabaja, ¿se otorga poder a las mujeres? \\
\hline
\end{tabular}


Caracterización del rol actual de la mujer en la gerencia de proyectos en Colombia.

\begin{tabular}{|c|c|}
\hline 28 & $\begin{array}{l}\text { Si la respuesta a la anterior pregunta es afirmativa, por favor, explique ¿cómo se evidencia este otorgamiento de } \\
\text { poder? }\end{array}$ \\
\hline 29 & En la organización en que trabaja, ¿se promueve y anima a las mujeres para que sean líderes? \\
\hline 30 & ¿Por qué? \\
\hline 31 & Si pudiera escoger, ¿̇preferiría tener un jefe ...? \\
\hline 32 & ¿Por qué? \\
\hline 33 & $\begin{array}{l}\text { Cuando el gerente del proyecto es una mujer, ¿ percibe diferencias (en comparación con la situación en que el } \\
\text { gerente de proyectos es un hombre) en la actitud de...? }\end{array}$ \\
\hline 34 & ¿Qué diferencias identifica? \\
\hline \multicolumn{2}{|r|}{ Desempeño de la mujer en la gerencia de proyectos. } \\
\hline 35 & $\begin{array}{l}\text { En una escala de } 0 \text { a 100, indique el porcentaje de proyectos a su cargo en los que se ha cumplido con la totalidad } \\
\text { de los objetivos }\end{array}$ \\
\hline 36 & $\begin{array}{l}\text { En una escala de } 0 \text { a } 100 \text {, indique el porcentaje de proyectos a su cargo en los que se ha con cumplido con el } \\
\text { cronograma }\end{array}$ \\
\hline 37 & $\begin{array}{l}\text { En una escala de } 0 \text { a 100, indique el porcentaje de proyectos a su cargo en los que se ha cumplido con el } \\
\text { presupuesto }\end{array}$ \\
\hline 38 & $\begin{array}{l}\text { En una escala de } 0 \text { a 100, indique el porcentaje de proyectos a su cargo que considera exitosos de acuerdo con los } \\
\text { criterios de su organización }\end{array}$ \\
\hline 39 & $\begin{array}{l}\text { Según su percepción, ¿̇se detectan diferencias en cuanto al desempeño de los proyectos liderados por mujeres y } \\
\text { por hombres debido específicamente a la condición de género? }\end{array}$ \\
\hline 40 & $\begin{array}{l}\text { De acuerdo con su experiencia, ¿̇ué habilidades aplicadas a la gerencia de proyectos tienen más desarrolladas las } \\
\text { mujeres que los hombres? }\end{array}$ \\
\hline 41 & $\begin{array}{l}\text { En las organizaciones en que ha trabajado, ¿cómo evaluaría la aplicación de políticas de equidad de género } \\
\text { relacionadas con...? }\end{array}$ \\
\hline 42 & ¿Se promueve en la organización la participación activa de la mujer en roles de gerencia de proyectos? \\
\hline 43 & En la organización en que trabaja, ¿se reconocen diferencias entre hombres y mujeres en cuanto a...? \\
\hline 44 & $\begin{array}{l}\text { ¿En qué se ve reflejada (evidencias) la forma en que en la organización se reconocen diferencias entre hombres y } \\
\text { mujeres? }\end{array}$ \\
\hline 45 & $\begin{array}{l}\text { De acuerdo con su percepción, ¿̇tienen las mujeres y los hombres iguales oportunidades de ascender en la } \\
\text { organización? }\end{array}$ \\
\hline 46 & $\begin{array}{l}\text { ¿Siente que como mujer en comparación con los hombres está siendo efectivamente apoyada por la alta dirección } \\
\text { de la organización? }\end{array}$ \\
\hline 47 & ¿Por qué? \\
\hline 48 & $\begin{array}{l}\text { De acuerdo con la clasificación de proyectos en su organización, ¿̇manejan las mujeres proyectos de mayor o } \\
\text { menor complejidad que los hombres? }\end{array}$ \\
\hline 49 & $\begin{array}{l}\text { De acuerdo con la clasificación de proyectos en su organización, ¿manejan las mujeres proyectos de mayor o } \\
\text { menor costo que los hombres? }\end{array}$ \\
\hline 50 & ¿Siente que en la organización hay discriminación en contra de la mujer? \\
\hline 51 & ¿Se ha sentido víctima de la discriminación en el ejercicio de la gerencia de proyectos? \\
\hline \multicolumn{2}{|r|}{ Información general } \\
\hline 52 & Nombres y apellidos (opcional) \\
\hline 53 & Nombre de la empresa en que trabaja (opcional) \\
\hline 54 & Dirección de correo electrónico (opcional) \\
\hline
\end{tabular}

